\title{
Idiopathic posterior uveitis
}

INSERM

\section{Source}

INSERM. (1999). Orphanet: an online rare disease and orphan drug data base. Idiopathic posterior uveitis. ORPHA:280917

Idiopathic posterior uveitis is a rare, potentially sight-threatening, ocular disease, not attributed to any specific ocular or systemic cause, characterized by focal, multifocal or diffuse non-infectious inflammation in the posterior uvea (i.e. choroiditis, chorioretinitis, retinitis and neuroretinitis). Visual morbidity due to complications (including cystoid macular edema and choroidal neovascularization) has been reported. 